\title{
AN INTEGRATED APPROACH FOR OPTIMIZING THE SPATIAL PLANNING OF TOURIST POIS FOR TRAFFIC ACCESSIBILITY: DALIAN CITY, CHINA
}

\author{
QUNSONG ZHANG ${ }^{1,2}$, EUNYOUNY KIM ${ }^{1}$, CUIXIA YANG $^{2} \&$ FUCUN CAO $^{2}$ \\ ${ }^{1}$ School of Knowledge Science, Japan Advanced Institute of Science and Technology, Japan \\ ${ }^{2}$ Landscape Architecture, School of Art \& Design, Dalian Polytechnic University, China
}

\begin{abstract}
It is important for urban tourism development to focus on the accessibility of tourist points of interest (POIs). The spatial distribution of POIs should be closely linked to sustainable traffic development. In recent years, smartphones and global positioning systems have provided strong technical support for tourist POIs through social media. Many studies have been conducted to investigate the correlation between POIs and transportation from different perspectives. There is however a lack of quantitative research on the correlation between traffic accessibility and the distribution of urban tourist POIs using space syntax theory. This study proposes a method for optimizing the layout of tourist POIs based on traffic accessibility. We crawled 2,322 tourist POIs in Dalian as research objects, adopted kernel density estimation and constructed spatial syntax models. We analysed these models from the perspective of the spatial distribution characteristics of the POIs and traffic accessibility. The results showed no direct correlation between the spatial distribution of POIs and road networks. The conclusion is that the distribution of the most popular POIs does not coincide with the roads with the highest accessibility in Dalian. Therefore, we propose feasible optimization strategies for spatial planning of tourist POIs and sustainable traffic development.
\end{abstract}

Keywords: accessibility, POIs heat distribution, spatial syntax, sustainable traffic development, tourist point of interest

\section{INTRODUCTION}

\subsection{Research background}

Tourist cities, such as Dalian in China, need tourism points of interest (POIs) optimization for their sustainable development. Tourism space optimization refers not only to the distribution of tourism resources, but also to the transport networks serving those resources. This involves comprehensive land use planning and collaborative synergy with the government. Transportation is a vital economic contributor to satisfying tourism demand, and transportation costs have a significant influence on destination selection. Thus, the transportation network is an organic component linking tourist POIs that can affect the development of those POIs, positively or negatively. The degree of transportation convenience has become an important indicator of the effectiveness of regional economic development [1], [2] [3]. As tourism transportation is a significant driver of the development of mass transportation, coordinating and planning the spatial layout of tourist POIs is of great importance to the sustainable development of urban transportation.

In recent years, smartphones, global positioning systems (GPSs) and travel websites have started providing strong technical support for tourist POIs, with the help of social media [4], [5]. The correlation between POIs and transportation has been studied by several researchers, 
from different perspectives. Yue et al. [6] proposed a relationship between the distribution of POIs and the community. Zhang et al. [7] used POIs and real-time traffic volume to identify traffic jams and proved the lack of correlation between different land use types. The accessibility of POIs for the elderly and disabled, from a small-scale perspective has also been studied [8], [9]. Wang and Xu [10] analysed the road traffic density and spatial network formed by the tourist POIs. Israeli and Mansfeld investigated the interrelations between urban tourism and transportation systems from a large-scale perspective [11]. He et al. [12] studied the spatially stratified association between leisure entertainment activities and street configurations using the kernel density estimation (KDE) method. Sugimoto et al. [13] examined the relationship between visitor mobility and urban spatial structures through GPS tracking technologies and questionnaires. Li et al. [14] introduced an access measure of network KDE to compute accessibility to POIs constrained by road networks. Other researchers have evaluated public transport accessibility and taxi travel demand. They estimated traffic patterns by implementing a gravity model using a geographically weighted regression model and improving the centrality of the station index [15], [16], [17] However, there is a lack of quantitative research on the relationship between road accessibility and the distribution of urban tourist POIs using space syntax theory.

Based on the POI data from the Gaode Map application (AMAP) and OpenStreetMap, the current study uses geographic information system (GIS) visualization technology to analyse the spatial distribution characteristics of different types of tourist POIs in nine administrative districts of Dalian as well as the heat distribution characteristics of POIs. Through a combination of spatial syntax parameters and GIS positioning, the accessibility of POIs of different heat levels in Dalian was analysed, and optimization strategies for tourism and sustainable traffic development were proposed.

\subsection{Research area}

This study was conducted in Dalian, which is a central city in Liaoning Province, a port, and a scenic tourist city on the northern coast of China. It is situated on the southern edge of the Liaodong Peninsula, at the junction of the Huanghai Sea and the Bohai Sea, between $38^{\circ} 43^{\prime} \mathrm{N}, 120^{\circ} 58^{\prime} \mathrm{E}$ and $40^{\circ} 12^{\prime} \mathrm{N}, 123^{\circ} 31^{\prime} \mathrm{E}$. The total land area of the city is $12,573.85 \mathrm{~km}^{2}$, of which the urban area is $2,414.96 \mathrm{~km}^{2}$. The registered population is 5.952 million. There are many mountains and hills in the study area, with few plains and lowlands. The terrain is high and wide in the north and low and narrow in the south. The terrain slopes from the central axis to the sea areas on the southeast and northwest sides and is long and gentle on the side facing the Huanghai Sea. As of 2020, the city had jurisdiction covering seven districts, one county, and two county-level cities, as shown in Fig. 1. Zhongshan, Shahekou, Xigang and Ganjingzi districts form the city centre of Dalian, and Wafangdian and Zhuanghe are countylevel cities. As a city rich in natural resources for tourism, Dalian has a coastline of 2,211 km with many famous geological remains and landscapes. In addition, Dalian has a distinctive cultural landscape, with many historical heritage sites and a special urban landscape. Owing to special climatic factors, Dalian is rich in agricultural resources and has varied regional characteristics. In recent years, many leisure agricultural attractions have emerged in suburban areas. This study aims to examine the road-based transportation of Dalian; therefore, the study area does not include Changhai County. 


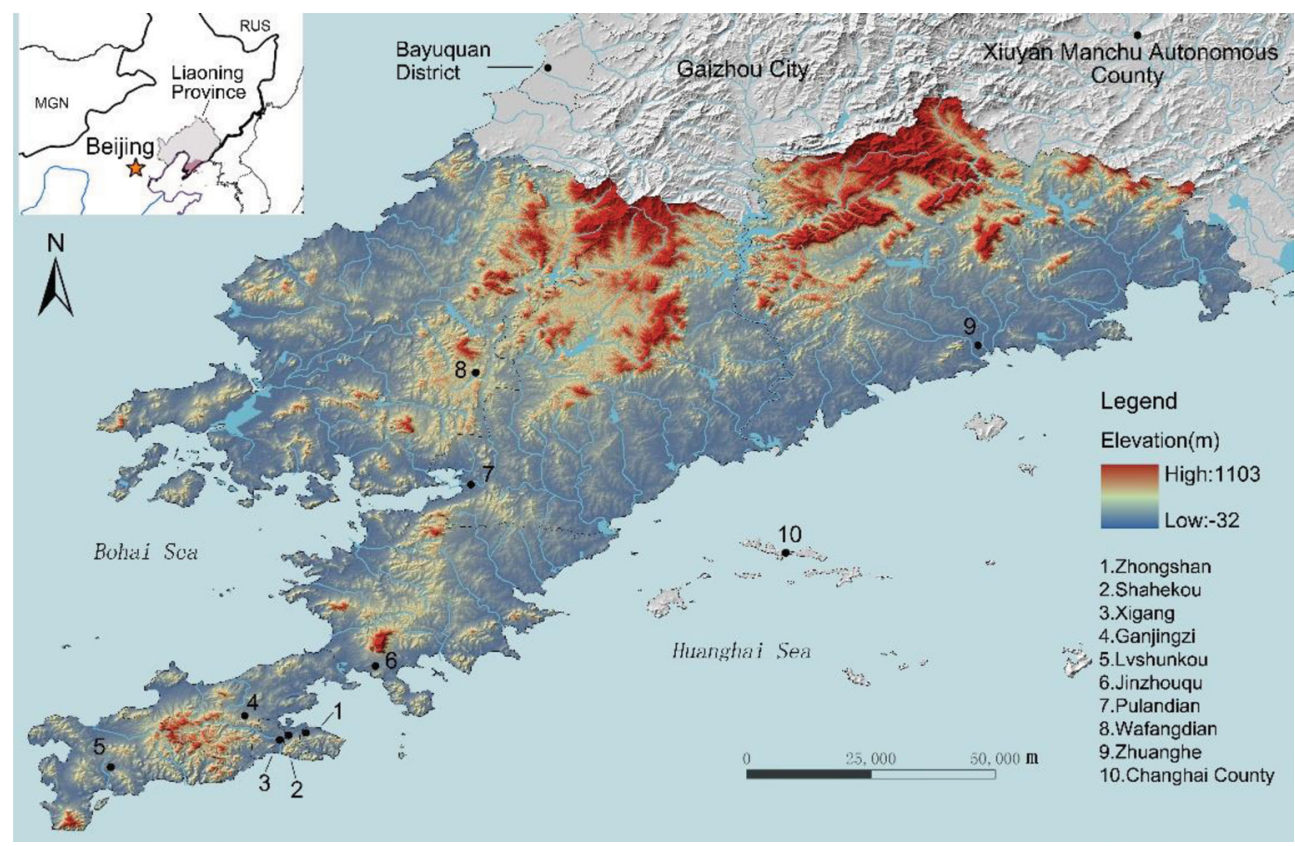

Figure 1: Study area and topographic map of study area.

\section{MATERIALS AND METHODS}

\subsection{Data collection}

Data collection was sourced mainly from AMAP, which is the most popular application used by Chinese residents for local travel. In addition, it is a platform for commenting and sharing information on POIs, mainly based on tripartite reviews. First, we obtained relevant POI data such as scenic spots, leisure farms and picking gardens, linked keywords to the data and then crawled the tourist POIs data of Dalian on the AMAP. The information obtained included latitude and longitude, name, address, number of comments, rating and types of POIs. After performing coordinate correction, screening, removal of duplicate data, and field investigation and verification of the data, a total of 2,322 POIs were collected by 30 October 2020. In addition, we divided tourist POIs into four categories: natural resources (I: 995), leisure agricultural (II: 469), commercial entertainment (III: 238) and humanistic resources (IV: 620). Using ArcGIS 10.2 software, we obtained the spatial distribution map of tourist POIs.

The basic road image was downloaded from OpenStreetMap, which contains multiple levels of roads. We then used AutoCAD software to draw axial node counts (7834), imported the Depthmap software and passed node detection. Subsequently, the results for each road syntax variable were output.

\subsection{Analysis methods}

\subsubsection{Space syntax theory}

Spatial syntax theory studies the degree of correlation between urban spatial structures and human social activities by quantitatively analysing the spatial environment [18]. The line 
segment method of space syntax further considers the accessibility of people and vehicles in the space flow based on the axis analysis method. The line segment method is used to aggregate and analyse the urban spatial structure and POI distribution using parameters such as the integration, connectivity, depth, choice, intelligibility and synergy.

Areas with a high degree of selection tend to have more traffic. The probability of the shortest path from a certain node space, $i$, to all other node spaces, $E_{\mathrm{i}}$, is calculated from Equation 1 :

$$
E_{i}=\frac{1}{(N-1)(N-2)} \sum_{j=k=1}^{N} \frac{n_{j, k}(i)}{n_{j, k}}
$$

where $N$ is the number of node spaces; $n_{j, k}(i)$ is the number of times the shortest path from node space $i$ to node $j$ is passed; $k$ is the number of other node spaces next to the node space; and $n_{j, k}$ is the total number of shortest paths in the system.

Connectivity $C_{i}$ is the connectivity of a certain node space, expressed by the number of node spaces adjacent to a certain node space. The higher the degree of connectivity, the better the spatial permeability of a certain node. $C_{i}$ is calculated from Equation 2:

$$
C_{i}=k(i) \text {. }
$$

We used the global integration degree $\left(I_{i}\right)$ to test the global accessibility of Dalian tourist POIs. The higher the integration degree, the stronger the concentration of a certain node space and the better the accessibility. $I_{i}$ is calculated from Equation 3:

$$
I_{i}=\frac{1}{A_{R R, i}}=\frac{E_{m}}{A_{R, i}}=\frac{m\left[\log _{2}\left(\frac{m+2}{3}-1\right)+1\right]}{(m-1)|D-1|},
$$

where $A_{R R, i}$ is the actual relative asymmetry, $A_{R, i}$ is the relative asymmetry, $E_{m}$ is the standard value of $A_{R, i}$ versus $A_{R R, i}$ after processing, and $m$ is the number of node spaces in the system.

\subsubsection{Kernel density estimation}

KDE reflects the law of distance attenuation. Setting different bandwidths changes the number and position of the identified centre positions. This study used the KDE method to identify the spatial distribution density and concentrated location of tourist POIs. In addition, the POI heat analysis reflects the trend of crowd gathering depending on the number of comments crawled on the AMAP.

\section{RESULTS}

\subsection{Spatial distribution characteristics of tourist POIs}

\subsubsection{Overall distribution of tourist POIs}

As shown in Fig. 2, there are significant differences in the distribution of POIs in Dalian from north to south. Zhongshan, Shahekou, Xigang and Lvshunkou formed several large-scale agglomeration areas. The distribution of POIs in Pulandian, Wafangdian and Zhuanghe is very scattered and primarily close to the coastal areas. However, there are many small-scale 


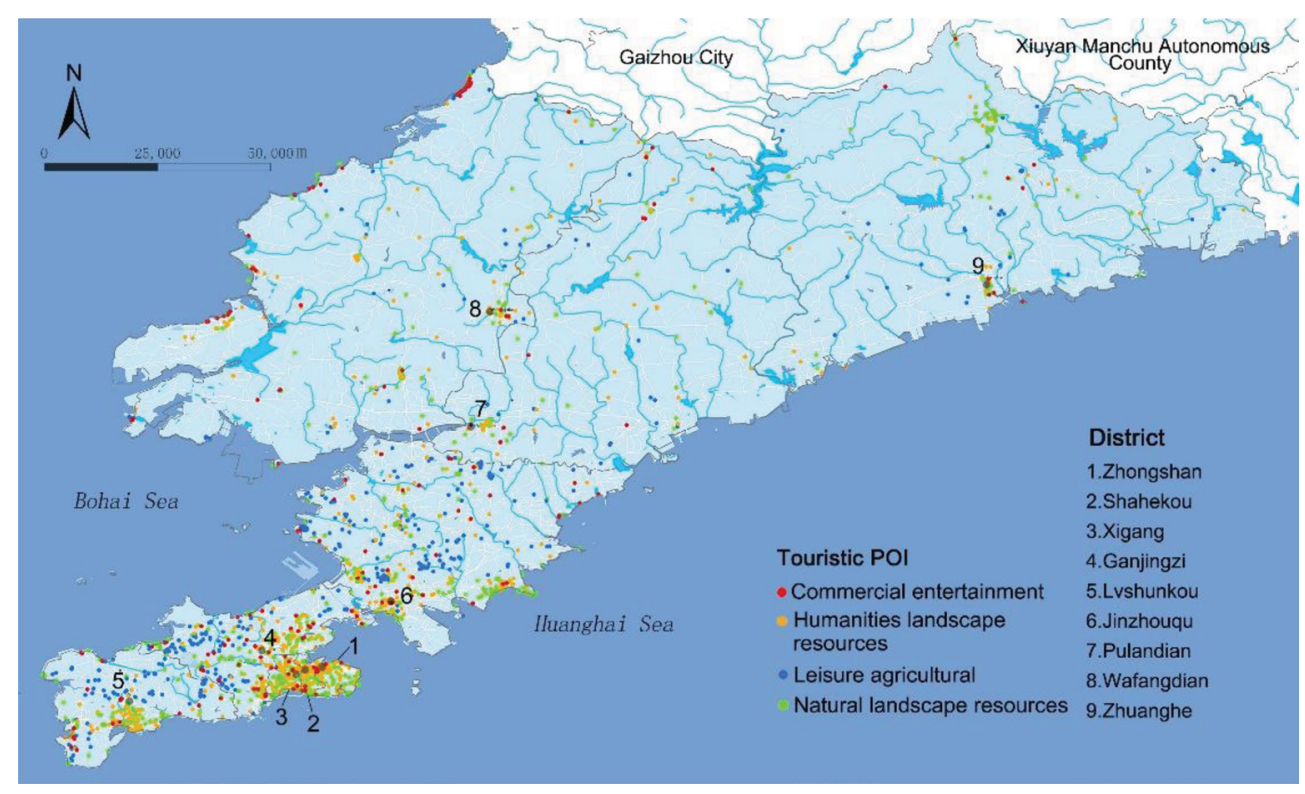

Figure 2: Distribution of tourist POIs in Dalian.

agglomeration areas in the southeast and west near the sea and downtown of Wafangdian. Generally, the POIs are in a group-type and non-uniform distribution, forming a divergent spatial pattern from the southwest to the northeast. From the statistical data, the distribution of POIs in each administrative district is also significantly different, showing obvious nonequal distribution characteristics. As shown in Table 1, Jinzhou and Ganjingzi have the largest number of POIs; the smallest are in Xigang. Ganjingzi, Jinzhou, Lvshunkou and Wafangdian account for $64.93 \%$ of the city's POIs number. Their spatial distribution is related to the size of the land area, the number of tourism resources and the degree of development in each district. The highest density of POIs is in Shahekou, and the lowest is in the Pulandian district.

\subsubsection{Spatial distribution pattern of different types of tourist POIs}

In this study, the tourist POIs involved include not only tourist attractions, but also entertainment venues and leisure agriculture. Tourist attractions can be divided into two types: natural resources and humanistic resources, depending on the reasons for tourism resources or their attributes. Natural tourist spots include natural landscapes composed of natural characteristics such as topography, climate, environment, animals and plants. Humanistic tourist spots include historical sites, religious buildings, art galleries, museums and architectural areas that represent the city's style. Such tourist areas represent the artistic development and cultural achievements of human activities. In addition, there is tourism connected with commercial entertainment, which provides significant recreation services. This type includes resorts, hot spring clubs, ski resorts, amusement parks and traditional trading areas. The last tourism category is leisure agriculture, which is the transformation of agriculture into a tertiary (service) industry and which has gradually developed in the past ten years in Dalian. It includes ecological sightseeing parks, picking gardens, and fishing parks.

After many experiments, four classified nuclear density grid maps were generated (see Fig. 3). Natural resources and humanistic resources tourism spots present an obvious 
Table 1: Distribution of tourist POIs in Dalian (Source: Statistical Yearbook of Liaoning Province, 2019.) Note: I: Natural tourist POIs; II: Leisure agricultural POIs; III: Commercial entertainment POIs; IV: Humanistic tourist POIs.

\begin{tabular}{lllllllll}
\hline District & $\begin{array}{l}\text { Touristic } \\
\text { POI }(\mathrm{N})\end{array}$ & Proportion & $\begin{array}{c}\text { I } \\
(\mathrm{N})\end{array}$ & $\begin{array}{l}\text { II } \\
(\mathrm{N})\end{array}$ & $\begin{array}{l}\text { III } \\
(\mathrm{N})\end{array}$ & $\begin{array}{l}\text { IV } \\
(\mathrm{N})\end{array}$ & $\begin{array}{l}\text { Area } \\
\left(\mathrm{km}^{2}\right)\end{array}$ & $\begin{array}{l}\text { Density of POI } \\
\left(\mathrm{N} / \mathrm{km}^{2}\right)\end{array}$ \\
\hline Zhongshan & 192 & $8.27 \%$ & 110 & 2 & 7 & 73 & 40.10 & 4.79 \\
Shahekou & 175 & $7.54 \%$ & 98 & 0 & 8 & 69 & 23.94 & 7.31 \\
Xigang & 149 & $6.43 \%$ & 93 & 0 & 3 & 53 & 34.71 & 4.29 \\
Ganjingzi & 349 & $15.04 \%$ & 134 & 95 & 38 & 82 & 451.52 & 0.77 \\
Lvshunkou & 273 & $11.75 \%$ & 107 & 74 & 17 & 75 & 512.15 & 0.53 \\
Jinzhou & 603 & $25.96 \%$ & 249 & 180 & 42 & 132 & 1352.54 & 0.45 \\
Pulandian & 112 & $4.82 \%$ & 49 & 17 & 10 & 36 & 2769.90 & 0.04 \\
Wafangdian & 283 & $12.18 \%$ & 67 & 62 & 85 & 69 & 3576.40 & 0.08 \\
Zhuanghe & 186 & $8.01 \%$ & 88 & 39 & 28 & 31 & 3655.70 & 0.05 \\
\hline
\end{tabular}
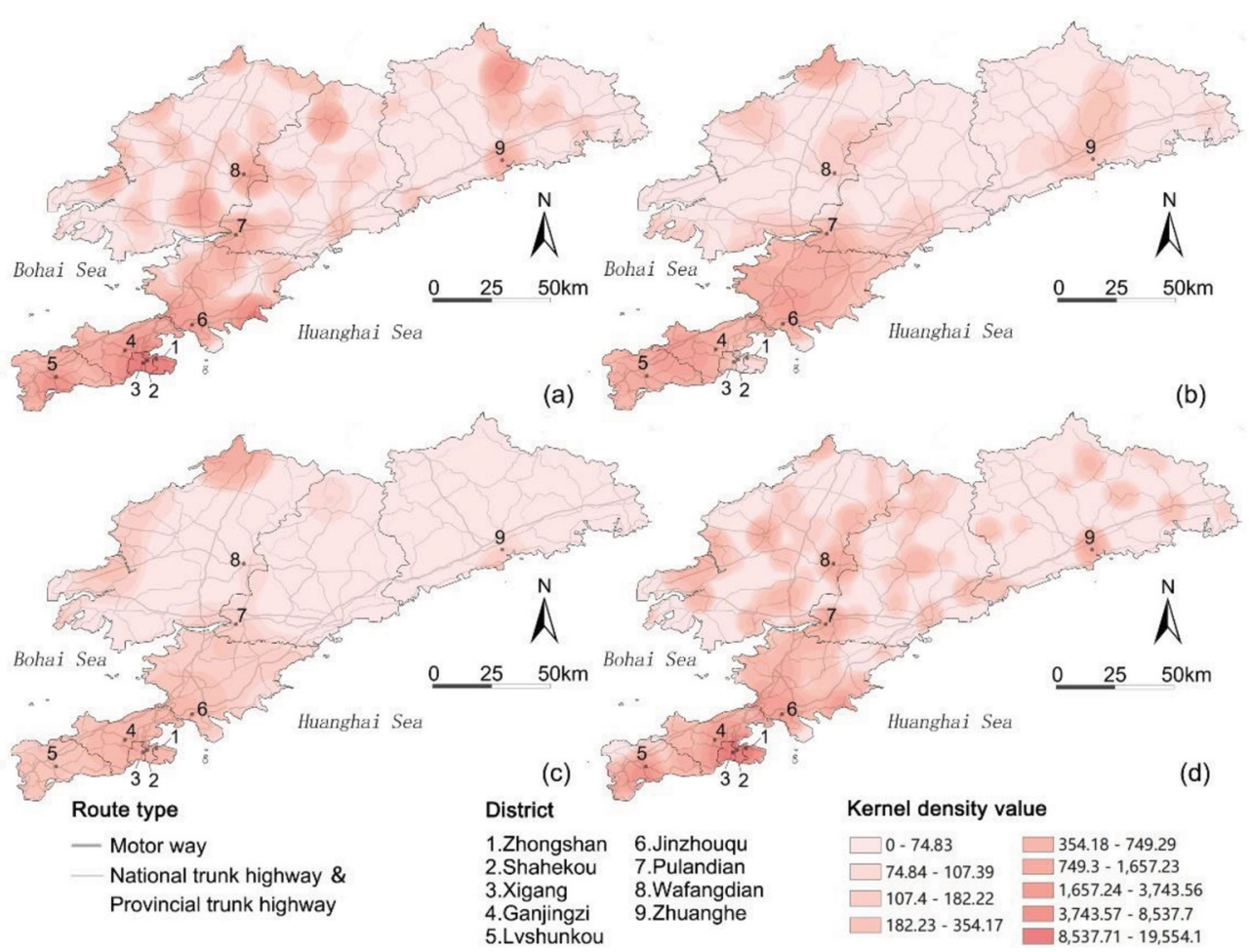

Figure 3: Kernel density of tourist POIs: (a) natural resources; (b) leisure agricultural; (c) commercial entertainment; (d) humanistic resources. 
multi-centre cluster distribution, most administrative districts and counties have a balanced proportion, as shown in Table 1 and Fig. 3(a) and 3(d). Natural resource tourist POIs are mainly concentrated in representative ocean parks and unique geological landscape scenic spots. Dalian is the only coastal landform in China; it has more than $30 \mathrm{~km}$ of sea erosion landforms. Meanwhile, the representative geological relic type of Bingyu National Geological Park in Zhuanghe is the quartz sandstone peak forest landform in the glacial period.

The tourist POIs of humanistic resources mainly revolve around the surrounding scenic spots. For example, there are several geological museums in the Jinshitan Scenic Area of the Jinzhou district. Most of the POIs at Lvshunkou are composed of historical sites and scenic spots in Port Arthur. Figure 3(c) shows that the commercial entertainment POIs are mainly composed of resort areas, hot springs, ski resorts and amusement parks. These POIs are often far away from the economically developed metropolis and agglomeration areas with extremely uneven spatial distribution. They appear in the three districts of the middle-west. Wafangdian and Pulandian have the highest number; however, there is no agglomeration area due to the large area covered by these districts, the low population density and the low distribution of scattered POIs. Figure 3(b) shows that the leisure agriculture POIs are mainly distributed in Jinzhou, Ganjingzi, Lvshunkou and the west side of Wafangdian. They also have the common characteristic of being far away from the metropolis. In general, the overall distribution of natural and humanistic resources tourist POIs is more even than that of commercial entertainment and leisure agriculture.

\subsubsection{Heat distribution of tourist POIs}

We took the total number of comments on the AMAP as heat values in the heat distribution map to measure the distribution of tourist flow and analyse the relatively unpopular POIs. Overall, as shown in Fig. 4, the two-level divergence of popularity is significant, and the number of comments on famous scenic spots that represent Dalian's cultural landscape is

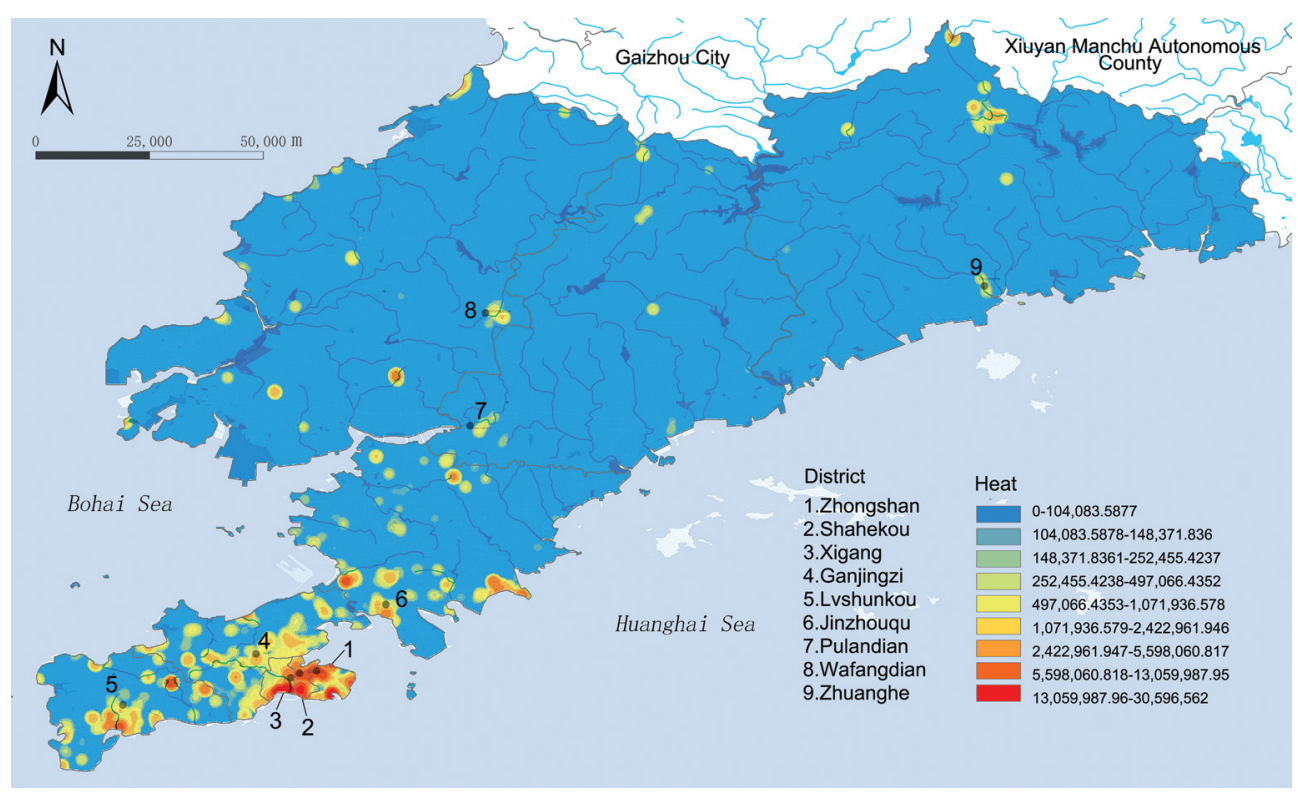

Figure 4: Heat of tourist POIs in Dalian. 
extremely high. By contrast, the number of comments on leisure agriculture and most resorts is extremely low; the spatial distribution is also extremely uneven.

The hottest areas are Zhongshan, Xigang and Shahekou, followed by Jinzhou, the coastal part of Lvshunkou and the west of Ganjingzi. The three northern districts are less important, consisting of scattered clusters. Figure 4 is consistent with Figs. 3(a) and 3(b) because the POIs with the higher heat are mostly composed of traditional scenic spots and humanistic landscapes with well-developed tourism resources.

\subsection{Analysis of accessibility of tourist POIs}

\subsubsection{Accessibility of tourist POIs in the global road network}

Through the analysis of the axial diagram of the road network in Dalian, as shown in Fig. 5(a), we can see that the higher part of the choice area is mostly composed of expressways and provincial roads. The axis with the highest degree of travel is located on the west side of Jinzhou; the axis with a higher degree of travel is evenly distributed on both sides of the peninsula. The overall road network is significantly affected by the terrain, and the effect of high-grade road networks is below the average. The overall choice and the level of connection with the highway needs to be improved further.

Figure 5(b) shows that the overall value of connectivity is low. The highest value appears in the Jinzhou area connecting the south-western end and the north of the peninsula. The gathering points of the road network coincide with the administrative centres of most districts. They are mainly concentrated in the central urban areas on the north side of Xigang, Zhongshan and Shahekou districts. In addition, some high-connectivity clusters are scattered in the southern part of Jinzhou district. The freeway road system was formed along both sides of

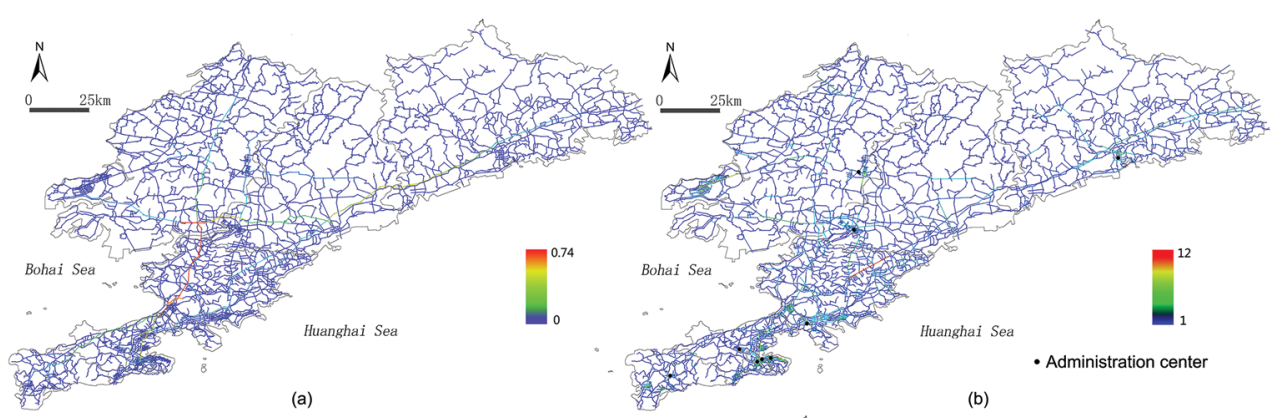

(a)

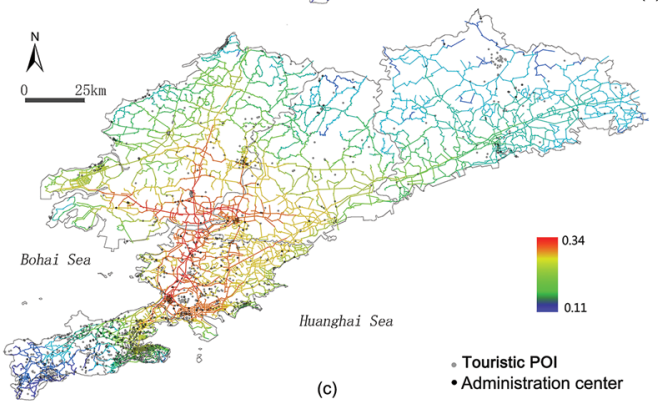

Figure 5: Space syntax variables of road network and tourist POI distribution in Dalian: (a) choice; (b) connectivity; (c) integration. 
the peninsula instead of a hierarchical structure spreading from the city centre to the suburbs, owing to topographical factors. The closed-loop road network is small, the connectivity is generally poor, and the road network's service capability is weak, resulting in weaker connectivity between the central city and suburbs.

Figure 5(c) shows that from the perspective of the choice and integration of the road axis, the road network of Dalian is generally in the form of a cross with the northwest part of Jinzhou and the junction of Wafangdian and Pulandian as the core. The central urban area and county central towns are secondary. The area with the highest concentration of tourist POIs does not coincide with the road network with the highest levels of integration. Moreover, the global integration of $39.2 \%$ of POIs is less than the average of all POIs $(0,225)$. Generally, the development of the road networks in areas with the densest tourist POIs distribution is uneven, and road network segments of different development levels are poorly connected. Most of the POIs in the city outskirts are in locations with low accessibility.

\subsubsection{Accessibility of heat of tourist POIs}

To investigate further the relationship between the differentiation of heat of tourist POIs in Dalian and the accessibility of road networks, we analysed the accessibility of different heat POI types within different buffer zones of the five levels of roads. According to the number of comments on the AMAP, the statistics of popularity are 17 very high, 38 high, 223 medium, 139 low and 1905 extremely low. The road axis of Dalian was divided into five grades, and multi-ring buffers were set for all road axes depending on the natural breaks (Jenks) method in the layer attribute classification method of the ArcGIS software [19]. As shown in Table 2, tourist POIs are mostly distributed in the buffer zone within $100 \mathrm{~m}$ of a fourth grade road, indicating that the overall accessibility of the tourist POIs in Dalian is moderate.

Table 2: Distribution of heat of tourist POIs for different road grades

\begin{tabular}{lllllll}
\hline Road grade & $\begin{array}{l}\text { Buffer zone } \\
(\mathrm{m})\end{array}$ & $\begin{array}{l}\text { Very high } \\
\text { heat }(\mathrm{N})\end{array}$ & $\begin{array}{l}\text { High } \\
\text { heat }(\mathrm{N})\end{array}$ & $\begin{array}{l}\text { Intermediate } \\
\text { heat }(\mathrm{N})\end{array}$ & $\begin{array}{l}\text { Low } \\
\text { heat }(\mathrm{N})\end{array}$ & $\begin{array}{l}\text { Very low } \\
\text { heat }(\mathrm{N})\end{array}$ \\
\hline First grade & $0-100$ & 0 & 2 & 15 & 18 & 0 \\
& $100-300$ & 1 & 3 & 10 & 3 & 48 \\
& $300-500$ & 0 & 0 & 5 & 2 & 22 \\
& $500-700$ & 0 & 2 & 7 & 2 & 18 \\
& $>700$ & 2 & 0 & 5 & 8 & 49 \\
Second grade & $0-100$ & 0 & 1 & 16 & 8 & 179 \\
& $100-300$ & 1 & 3 & 10 & 2 & 98 \\
& $300-500$ & 0 & 0 & 4 & 2 & 45 \\
& $500-700$ & 0 & 0 & 2 & 3 & 9 \\
& $>700$ & 1 & 2 & 4 & 5 & 85 \\
Third grade & $0-100$ & 4 & 6 & 26 & 12 & 183 \\
& $100-300$ & 5 & 2 & 21 & 11 & 137 \\
& $300-500$ & 0 & 2 & 8 & 3 & 38 \\
& $500-700$ & 0 & 0 & 2 & 2 & 22 \\
& $>700$ & 0 & 0 & 2 & 1 & 26 \\
\hline
\end{tabular}


Table 1: (Continued)

\begin{tabular}{lllllll}
\hline Road grade & $\begin{array}{l}\text { Buffer zone } \\
(\mathrm{m})\end{array}$ & $\begin{array}{l}\text { Very high } \\
\text { heat }(\mathrm{N})\end{array}$ & $\begin{array}{l}\text { High } \\
\text { heat }(\mathrm{N})\end{array}$ & $\begin{array}{l}\text { Intermediate } \\
\text { heat }(\mathrm{N})\end{array}$ & $\begin{array}{l}\text { Low } \\
\text { heat }(\mathrm{N})\end{array}$ & $\begin{array}{l}\text { Very low } \\
\text { heat }(\mathrm{N})\end{array}$ \\
\hline Fourth grade & $0-100$ & 1 & 7 & 39 & 7 & 254 \\
& $100-300$ & 1 & 2 & 14 & 15 & 123 \\
& $300-500$ & 0 & 0 & 5 & 2 & 57 \\
& $500-700$ & 0 & 0 & 0 & 1 & 28 \\
& $>700$ & 0 & 1 & 2 & 0 & 49 \\
Fifth grade & $0-100$ & 0 & 1 & 11 & 16 & 183 \\
& $100-300$ & 0 & 0 & 8 & 9 & 146 \\
& $300-500$ & 0 & 4 & 6 & 4 & 34 \\
& $500-700$ & 1 & 0 & 0 & 0 & 42 \\
& $>700$ & 0 & 0 & 1 & 3 & 29 \\
\hline
\end{tabular}

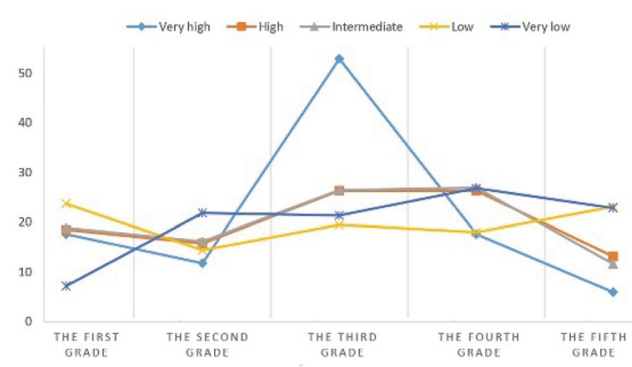

(a)

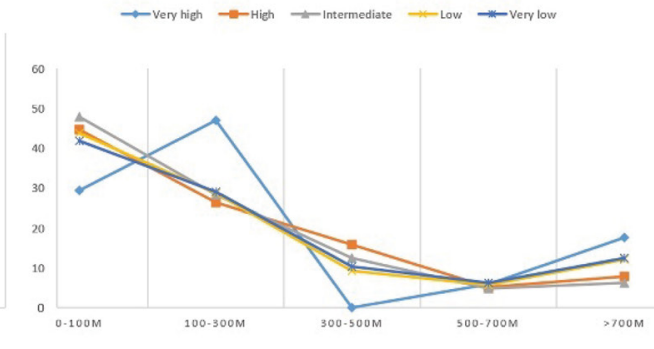

(b)

Figure 6: Heat of POIs by road grade and buffer zone:(a) road grades; (b) buffer zones.

Meanwhile, as shown in Fig. 6, although the high heat POIs are in areas with the lowest proportion of the overall population, mostly concentrated on third-grade roads and in the range 100-300 m distance from the road, the overall accessibility is not high. The proportion of road grade distribution of high and intermediate POIs is almost the same; very low heat POI is located near first grade roads, which have the lowest proportion, whereas fifth grade roads has the highest proportion. The lower the heat, the worse the accessibility. This is in line with the results of our previous studies; even the comments relating to some unpopular scenic spots show that the navigation route is wrong. Figure 6(b) shows that except for the hottest POIs, the proportions of the other four levels of POIs are close, with most of them being concentrated in the 100-300 m buffer zone. In addition, as shown in Fig. 4 and Fig. $5(\mathrm{c})$, there are different degrees of dislocation between areas with high heat and areas with high integration of the road. The most popular scenic spots are not only unevenly distributed, but also have poor accessibility. There are no popular tourist POIs near roads with high accessibility, which leads to varying degrees of waste of transportation resources and tourism resources. 


\section{DISCUSSION AND CONCLUSIONS}

Summarizing the discussion in the aforementioned section, the results indicate that the attraction of tourist POIs in Dalian is unbalanced. The resource development of the four types of tourist POIs is also uneven. The hottest and densest POI regions are not consistent with the most integrated regions, and most POIs have poor accessibility. Specific optimization strategies based on these problems are optimization of tourist POI resources and optimization of urban sustainable transport development.

\subsection{Optimization strategies of tourist POI resources}

Natural and historical resources constitute the unique cultural landscape of Dalian. Natural resources include marine and geological resources. Because of the coastal nature and characteristic geological structure of the area, a relatively complete transportation system has been formed around the famous Tiger Beach Ocean Park and Golden Pebble Beach Park. The proximity of these development sites to the metropolitan area of Dalian in space-time makes it easier for tourists to visit. However, other coastal landscapes are distributed around cities with low levels of integration, especially the renowned Bingyugou Geopark, which is far from the urban centre. Its accessibility is very low, resulting in fewer opportunities for tourists to visit. Relevant government departments should plan compound-type high-capacity tourist POIs with geoparks as the main attraction, leisure agriculture and commercial entertainment as supplements. High service quality and popularity will ensure repeat visits. Other POIs of natural resources that are far from the urban area and with low degrees of aggregation could also be integrated with different types of resources. For example, Minghu Resort could integrate several recreational services for different seasons, with skiing in winter, hiking in summer and hot spring hotels open all year round. Natural resources and leisure agriculture can also be combined organically. The Longwangtang Cherry Blossom Garden in the Lvshunkou district, which has the worst transportation infrastructure, and the Hengshan Temple Scenic Area could be integrated; this could increase customers both for the temple and the nearby picking gardens.

Humanistic resources POIs including the historical culture centre of Lvshunkou district and the modern art attractions for youth are all highly popular. The integration of cultural resources and commercial entertainment resources can not only increase the level of popularity of these centres, but also promote the development of cultural tourism. Generally, POIs where an integration centre is located should be strengthened. Meanwhile, the surrounding tourist attractions should be integrated to form multi-functional compound tourist POIs. This will allow tourists to make fewer trips and enrich the content of their activities. Tourist POIs should be optimized to ensure the sustainable development of transportation.

\subsection{Optimization strategies of urban sustainable transport development}

\subsubsection{Government regulation and planning}

Although private car ownership is growing rapidly in China, the government needs to avoid reducing the access to public transport. Transit-oriented developments around rail transit stations service urban spaces with high density and existing high levels of mixed walking, cycling, and public transportation. They can also be used to service areas with relatively dense tourist POIs and poor accessibility. Such developments would not only improve the proportion of urban public transport travel, but also promote the expansion of the tourism industry along urban rail transit routes and enhance urban vitality and the cultural image. For 
example, humanistic scenic spots in Lvshunkou and the coastal resort area in Wafangdian should strengthen the north-south connection and extend the subway lines and high-level city ring lines to increase traffic flow. Our research results indicated that the locations of some less popular tourist POIs are very remote. Poor roads should not be the reason for tourists being unable to reach such tourist POIs. Authorities should strengthen the development and management of roads around potential scenic spots. The development of POIs should be done in conjunction with the development of convenient transportation infrastructure. Motorways, national trunk highways and provincial trunk highways should be used as main roads, and rural roads should provide the links, to avoid wasting transportation resources. This applies, for example, to the leisure agricultural areas in the three northern districts of Dalian. Tourism organizations should use their own natural appeal and resources to strengthen the interaction and coordination with surrounding areas.

\subsubsection{Intelligent public transport services}

Traditional public transportation faces stiff competition from multiple transportation modes. With the development of information technology, increased usage of mobile phones, and big data, major Chinese cities have launched numerous shared mobility modes, such as online car-hailing services in 2016. New transport service requirements have been proposed. Big data should be used to integrate and share transportation resources; different transportation modes should be given the opportunity to complement and promote each other. The public welfare attributes of conventional buses should be transformed into "customized public transport" with market-oriented attributes.

Customized public transportation lines can be added for POI areas with high heat in remote locations, such as the Bingyugou Geopark, which are less accessible. For some popular POIs, such as the Forest Zoo in Zhongshan district, shuttle buses should be set up to form a complex transportation network. On the one hand, the goal should be to reduce private car travel as much as possible. On the other hand, accessibility by public transport using the direct route to popular POIs needs to be made convenient and attractive to avoid congestion.

\subsection{Conclusion}

Quantitative research on the spatial distribution of tourist POIs in nine districts of Dalian, in China, based on GIS and space syntax theory is feasible. The results can provide the basis for the sustainable development of urban traffic, POI spatial planning and development, and other aspects. However, we did not consider the impact of traffic trip volume and urban land use indicators owing to the difficulty of collecting data. There might be limitations in exploring traffic accessibility and the integration of scenic spots. Research should be conducted on local accessibility, especially traffic congestion in popular scenic spots and road convenience in unpopular scenic spots. In the future, other relevant indicators will be cited to further study the relationship between road sustainable development and various land use patterns.

\section{REFERENCES}

[1] Kovačić, M. \& Milošević, T., Interdependence of Transport and Tourism, Journal of Maritime \& Transportation Science, 52(1), pp. 99-111, 2016.

[2] Van Truong, N. \& Shimizu, T., The effect of transportation on tourism promotion: Literature review on application of the Computable General Equilibrium (CGE) Model, Transportation Research Procedia, 25, pp. 3096-3115, Elsevier B.V. 2017. 
[3] Ford, A., Barr, S., Dawson, R. \& James, P., Transport Accessibility Analysis Using GIS: Assessing Sustainable Transport in London, ISPRS International Journal of GeoInformation, 4(1), pp. 124-149, 2015.

[4] Naramski, M. \& Herman, K., The development of mobile tourism in the Upper Silesian metropolitan area of Poland, Sustainability (Switzerland), 12(1), pp. 1-22, 2020.

[5] Chantre-Astaiza, A., Fuentes-Moraleda, L., Muñoz-Mazón, A. \& Ramirez-Gonzalez, G., Science mapping of tourist mobility 1980-2019. Technological advancements in the collection of the data for tourist traceability, Sustainability (Switzerland), 11(17), pp. 1-32, 2019.

[6] Yue, Y., Zhuang, Y., Yeh, A.G.O, Xie, J.-Y., Ma, C.L. \& Li, Q.Q., Measurements of POIbased mixed use and their relationships with neighbourhood vibrancy, International Journal of Geographical Information Science, 31(4), pp. 658-675, 2017.

[7] Zhang, T., Sun, L., Yao, L. \& Rong, J., Impact analysis of land use on traffic congestion using real-time traffic and POI, Journal of Advanced Transportation, 2017, 2017.

[8] Jamaludin, M. \& Kadir, S. A., Accessibility in Buildings of Tourist Attraction: A case studies comparison, Procedia - Social and Behavioral Sciences, 35, pp. 97-104, 2012.

[9] Fatima, K., Moridpour, S., Saghapour, T. \& de Gruyter, C., Comparison of elderly public transport accessibility indices: time-based methods, Australasian Transport Research Forum, ATRF 2019 - Proceedings, 2019.

[10] Wang, Y. \& Xu, L., Analysis of urban points of interest and urban traffic from Shenzhen urban space optimization, IOP Conference Series: Earth and Environmental Science, 424(1), 2020.

[11] Israeli, Y. \& Mansfeld, Y., Transportation accessibility to and within tourist attractions in the Old City of Jerusalem, Tourism Geographies, 5(4), pp. 461-481, 2003.

[12] He, S., Yu, S., Wei, P. \& Fang, C., A spatial design network analysis of street networks and the locations of leisure entertainment activities: a case study of Wuhan, China, Sustainable Cities and Society, 44, pp. 880-887, 2019.

[13] Sugimoto, K., Ota, K. \& Suzuki, S., Visitor mobility and spatial structure in a local urban tourism destination: GPS tracking and network analysis, Sustainability, 11(3), pp. 919, 2019.

[14] Li, Q., Zhang, T., Wang, H. \& Zeng, Z., Dynamic accessibility mapping using floating car data: a network-constrained density estimation approach, Journal of Transport Geography, 19(3), pp. 379-393, 2011.

[15] Li, M., Kwan, M. P., Wang, F. \& Wang, J., Using points-of-interest data to estimate commuting patterns in central Shanghai, China, Journal of Transport Geography, 72, pp. 201-210, 2018.

[16] Liu, X., Sun, L., Sun, Q. \& Gao, G., Spatial variation of taxi demand using GPS trajectories and POI data, Journal of Advanced Transportation, 2020, 2020.

[17] Yang, R., Liu, Y., Liu, Y., Liu, H. \& Gan, W., Comprehensive public transport service accessibility index-a new approach based on degree centrality and gravity model, Sustainability (Switzerland), 11(20), 2019.

[18] Liu, C. \& Yu, R., Spatial accessibility of road network in Wuhan metropolitan area based on spatial syntax, Journal of Geographic Information System, 4, pp. 128-135, 2012.

[19] North, M. A., A method for implementing a statistically significant number of data classes in the Jenks algorithm, 6th International Conference on Fuzzy Systems and Knowledge Discovery, FSKD 2009, 1, pp. 35-38, 2009. 